\title{
Switching of oral anticoagulants in patients with non-valvular atrial fibrillation: A narrative review
}

\author{
Adane Kefale ${ }^{1}$, Gregory Peterson ${ }^{1}$, Woldesellassie Bezabhe ${ }^{1}$, and Luke Bereznicki ${ }^{1}$ \\ ${ }^{1}$ University of Tasmania
}

July 6, 2021

Approval of direct-acting oral anticoagulants (DOACs) for stroke prevention in atrial fibrillation (AF) was an important milestone, providing widened treatment choices along with the possibility for inter-drug switching after initiation. In addition to improved utilisation of oral anticoagulants (OACs) for stroke prevention, reports of switching among OACs are growing in the literature. Switching may influence clinical outcomes, healthcare costs and patient satisfaction. This review aimed to summarise the current literature on the pattern of OAC switching in patients with AF, including reasons for switching and clinical consequences following switching. We included articles published after 2013, following the introduction of apixaban; searched on June 27, 2020 from PubMed, Scopus and Embase. The review found that switching among OACs was common in clinical practice, significantly varying with the type of OAC. Studies reporting the reason for switching and clinical outcomes were comparatively limited. The reasons were often related to safety issues, poor anticoagulation control and ease of use. Factors that can increase the risk of bleeding and stroke were found to be associated with switching from vitamin $\mathrm{K}$ antagonists, but less for DOAC switching. Findings regarding bleeding outcomes following switching were inconsistent, possibly confounded with the type of OAC, reasons for switching and switching protocol. Despite the limited number of studies included and their relatively short follow-up periods, our review revealed that switching had minimal impact on stroke and other related thrombotic outcomes. Further prospective studies are needed to better understand possible reasons for switching and its influence on clinical outcomes. 